Article

\title{
Internet of Things (IoT) as Sustainable Development Goals (SDG) Enabling Technology towards Smart Readiness Indicators (SRI) for University Buildings
}

\author{
Ignacio Martínez ${ }^{1, *(\mathbb{D})}$, Belén Zalba ${ }^{1, * \mathbb{D}}$, Raquel Trillo-Lado ${ }^{1}$, Teresa Blanco ${ }^{1} \mathbb{D}$, David Cambra ${ }^{2}$ \\ and Roberto Casas ${ }^{1}$ (D) \\ 1 Aragon Research Engineering Institute (I3A) of University of Zaragoza (UZ), 50013 Zaragoza, Spain; \\ raquelt1@unizar.es (R.T.-L.); tblanco@unizar.es (T.B.); rcasas@unizar.es (R.C.) \\ 2 Green Office of University of Zaragoza (UZ), 50009 Zaragoza, Spain; dcambra@unizar.es \\ * Correspondence: imr@unizar.es (I.M.); bzalba@unizar.es (B.Z.)
}

check for updates

Citation: Martínez, I.; Zalba, B.; Trillo-Lado, R.; Blanco, T.; Cambra, D.; Casas, R. Internet of Things (IoT) as Sustainable Development Goals (SDG) Enabling Technology towards Smart Readiness Indicators (SRI) for University Buildings. Sustainability 2021, 13, 7647. https://doi.org/ $10.3390 /$ su13147647

Academic Editor: Antonio Caggiano

Received: 31 May 2021

Accepted: 6 July 2021

Published: 8 July 2021

Publisher's Note: MDPI stays neutral with regard to jurisdictional claims in published maps and institutional affiliations.

Copyright: (c) 2021 by the authors. Licensee MDPI, Basel, Switzerland. This article is an open access article distributed under the terms and conditions of the Creative Commons Attribution (CC BY) license (https:// creativecommons.org/licenses/by/ $4.0 /)$.

\begin{abstract}
Non-residential buildings contribute to around 20\% of the total energy consumed in Europe. This consumption continues to increase globally. Smart building proposals (focused on Nearly Zero Energy Building (NZEB), air quality monitoring, energy saving with thermal comfort, etc.) were already necessary before 2020, and the pandemic has made this research and development area more essential. Furthermore, the need to meet the Sustainable Development Goals (SDG) and obtain technological solutions based on the Internet of Things (IoT) requires holistic contributions through real installations that serve as spaces for measuring, testing, study and research. This article proposes a "measure-analyse-decide and act" methodology to quantify the Smart Readiness Indicator (SRI) for university buildings as a reference environment for energy efficiency and COVID-19 prevention models. Two conceptual spaces (physical and digital) within two dimensions (users and infrastructures) are designated over an IoT three-level model (information acquisition, interoperable communication, and data-driven decision). An IoT ecosystem (sensoriZAR) was implemented as a proof-of-concept of a smart campus at the University of Zaragoza, Spain. Focused on $\mathrm{CO}_{2}$ and energy consumption monitoring, the results showed effectiveness through real installations, demonstrating the IoT potential as SDG-enabling technologies. These contributions allow not only experimental lab tests (from the authors' expertise in several specialties of Industrial, Mechanical, Design, Thermal, Electrical, Electronic, Computer and Telecommunication Engineering) but also a reference model for direct application in academic works, research projects and institutional initiatives, extendable to professional environments, buildings and cities.
\end{abstract}

Keywords: Internet of Things (IoT) for Sustainable Development Goals (SDG); university campus as living lab; energy efficiency building; Nearly Zero Energy Building (NZEB); digital twins; smart building technologies; Smart Readiness Indicator (SRI); service design thinking; educational spaces

\section{Introduction}

Non-residential buildings (e.g., public institutions, industrial factories, government buildings) contribute to around $20 \%$ of the total energy consumed in Europe, according to a 2020 special report by the European Court of Auditors. This consumption continues to increase globally [1]. Non-residential buildings, given their savings potential, should have priority consideration in the European Union (EU) 2030 agenda to meet the Sustainable Development Goals (SDGs) with the commitment to reduce energy consumption by more than $20 \%$ by 2020 and $32.5 \%$ by 2030 [2]. The EU says in its "Renovation wave for Europe" strategy [3] that a trend of renewal for Europe allows "greening our buildings, creating jobs, and improving our lives". Its goal is to double the annual energy renewal rates in the next ten years. In 2014-2020, the EU allocated approximately $€ 14$ billion to improving the energy efficiency of buildings. In addition, member states budgeted $€ 5.4$ billion in national 
co-financing for the improvement of all types of buildings. These renovations will improve the quality of life for the people who live in or/and use buildings, reduce greenhouse gas emissions and create up to 160,000 additional green jobs in the construction sector. Therefore, energy efficiency in buildings is becoming a determining factor for investment and financing in our environment. Furthermore, this strategy is reinforced with the action plan "European Green Deal": an agreement to promote the efficient use of resources towards a clean circular economy, restore biodiversity and reduce pollution [4]. The plan describes how to obtain a just and inclusive transition for the EU to be climate neutral by 2050, following the proposal of the "European Climate Law" to turn this political commitment into a legal obligation [5].

In the last 20-30 years, notable advances have been made in building energy simulation, but much less progress has been made in terms of measuring, analysing and learning about the real operation of the building-facilities integration. Therefore, a contributor toward these objectives is the use of continuous improvement cycles based on the "measure-analyse-decide and act" methodology [6]. Thanks to analytical procedures for measurement and data-driven decisions, the corresponding audits of energy parameters in buildings can be carried out to achieve compliance with national and international regulations, meet goals established in the SDGs, and propose cost-effective solutions, such as the one presented in this article.

The proposed methodology aligns with the Strategy for Energy Rehabilitation in the Building Sector in Spain (ERESEE) [7], which proposes a logical order for the actions: (1) reduce demand through energy efficiency actions, (2) use highly efficient systems and (3) provide the final energy through renewable energies, mainly produced in situ. Figure 1 shows the main technological advances and tools necessary to move towards climate neutrality in the building sector from 2030 and reach a zero-emissions level by 2050 . Following the ERESEE strategy, each milestone in Figure 1 occupies a circle, located on the date of its maturity and proportional to its contribution. Moreover, the grey circles can be considered information technology (IT) subareas, showing the need to monitor the facilities to quantify their performance and efficiency before and after the actions. To demonstrate the success of the improvements, data comparisons, quantitative conclusions and the publication of results are essential to replicate the improvements in multiple areas.

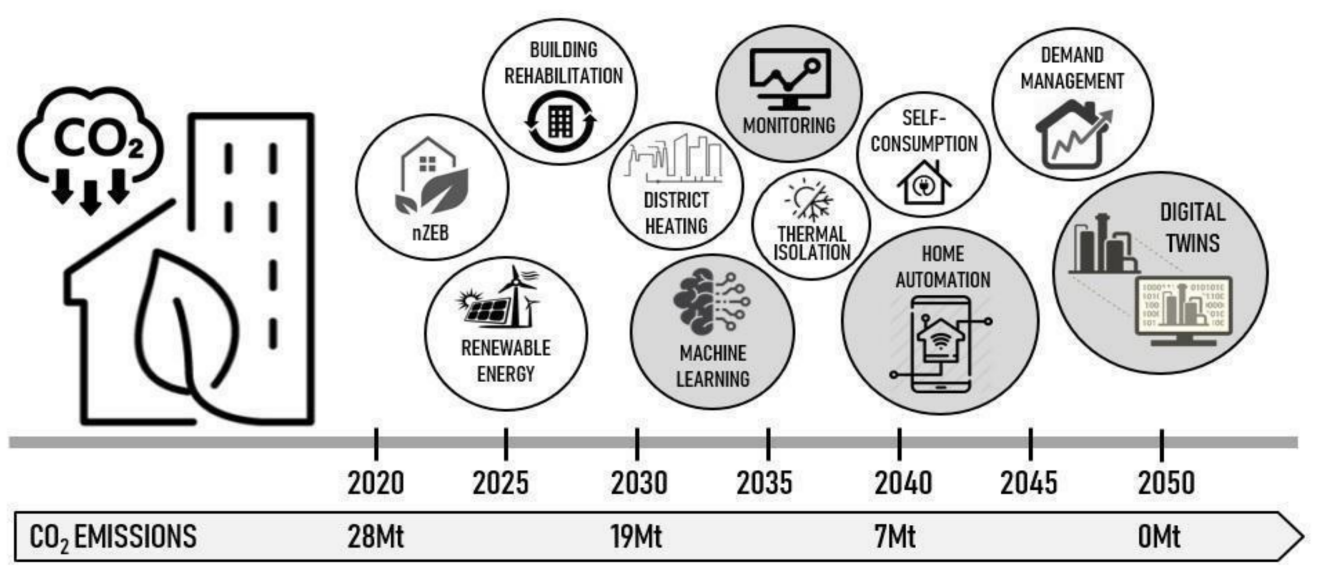

Figure 1. ERESEE strategy. Milestones towards climate neutrality, adapted from [7].

At present, the proposal for cost-effective solutions developed on data-based decisionmaking involves technologies based on the Internet of Things (IoT) preferably [8]. IoT is one of the technological paradigms destined to exponentially increase the connectivity of various devices. A main strength of IoT is its high impact on the daily behaviour of potential users [9]. The unstoppable growth of IoT devices (currently more than 30 billion connected in the world) provides an incalculable amount of data that, if correctly transmitted and processed (through cloud computing and similar technologies), will turn into 
usable information [10]. This information, conveniently stored, visualised and analysed (using Artificial Intelligence, Machine Learning, Process Mining, etc.), will generate knowledge that can produce positive feedback in the acquisition and processing of the data and intelligent decision-making based on valuable data [11]. According to the United Nations Educational, Scientific and Cultural Organization (UNESCO), sustainable and innovative solutions and systems require efficient, transparent and dynamic scientific efforts [12]. The objectives are industrial and commercial technological development and innovation guaranteeing the protection and confidentiality of data; and promoting the open availability of data such that third parties can offer valuable services, especially technological innovation and open science in university and research environments [13].

Following the first two points of the ERESSE strategy (demand reduction and performance increase), a relevant element is the ventilation load (which constitutes between $30 \%$ and $70 \%$ of demand). The $\mathrm{CO}_{2}$ measurement is an essential factor in the current COVID-19 pandemic and a key parameter for Demand-Controlled Ventilation (DCV) as a smart ventilation strategy [14]. Natural ventilation systems (simply, the opening of windows) do not allow adequate control over the entrance of outside air, making it difficult to determine if the ventilation flow is meeting the needs of the indoor environment. In addition, natural ventilation eliminates the potential use of filtration systems' heat recovery systems with thermal energy. Given that university buildings are characterised by significant intermittency or variability in levels of occupancy, it is crucial to improve the performance of the mechanical ventilation automation and control system, adapting the outdoor air flows to the actual occupation of each premises, or thermal zones [15]. Therefore, the most appropriate control involves sensors that measure parameters of indoor air quality $\left(\mathrm{CO}_{2}\right)$ [16]. This improvement measure is expected to significantly impact university building rehabilitation plans.

Since this research area is an emerging field, the available literature is still limited. Nonetheless, some related words can be highlighted. In [17], assuming this scope is a relatively new development, a comprehensive review was detailed by analysing the potential of connecting Building Information Modelling (BIM) and smart buildings with IoT-based data sources. In [18], several examples of IoT implementation (within the last five years) in residential and commercial buildings were reviewed. Compared to the reviewed literature, the approach in this paper advances in the integration of open-source tools, open data and open hardware with ultra-low consumption technologies, such that this knowledge can be available to the population. Even so, several works have been published on open-source IoT devices in academic buildings. In [19], a hardware IoT infrastructure was proposed for monitoring school buildings in Greece. A study by [20] developed an IoT lab system to monitor the overall activities of the lab. The differential value of the contributions of this paper is that the entire buildings (not only researching labs) become an experimental testbed for the teaching/learning of current and future generations. Thus, the methodology proposed constitutes a reference model for direct application in academic works, researching projects and institutional initiatives, extendable to professional environments, healthy buildings and cities.

Finally, to promote energy efficiency through smart building technologies and following the European Energy Performance of Buildings Directive (EPBD) [21], a key element is the Smart Readiness Indicator (SRI) [22]. This indicator qualifies the capacity of a building (or building unit) to adapt its general performance to the occupant needs (also optimising energy efficiency) and to allow energy flexibility in the performance according to the network parameters. SRI is essential to make building owners and occupants aware of the value hidden in building automation and the digital supervision of building technical systems, providing considerable savings thanks to the new intelligent functionalities [23]. Previous work on the application of the SRI has been sought. Only one has been found [24], the authors evaluated the SRI of two buildings and detected some weak points in the methodology. This paper advances in this line, evaluating the SRI of the 
university buildings and especially taking advantage of this useful tool to propose ideas for retrofitting actions.

Smart building proposals, focused on Nearly Zero Energy Buildings (NZEBs), air-quality monitoring, energy savings with thermal comfort, etc., were necessary before 2020, and the pandemic has made this research and development area more essential. Therefore, cost-effective and scalable solutions are required to apply smart building technologies in different contexts, encompassing global scenarios. This article proposes a "measure-analyse-decide and act" methodology to quantify the SRI from a holistic perspective. The research group includes architecture and several specialities of industrial, mechanical, design, thermal, electrical, electronic, computer and telecommunication engineering. This transversal perspective is intended to serve as a reference environment of measurements, testing, study and research for energy efficiency and COVID-19 prevention models. The Section 2 details two conceptual spaces (for users and infrastructures, within two dimensions (physical and digital), over an IoT three-level model (information acquisition, interoperable communication, and data-driven decision). The Section 3 shows several success stories, focused on $\mathrm{CO}_{2}$ and energy consumption monitoring, through real installations as a proof-of-concept of IoT as SDG-enabling technologies. Finally, the Section 4 contains a critical discussion that supports the Section 5 and proposes further studies.

\section{Materials and Methods}

Learning implies a performance: a factory (from Latin, facere). The university environment has traditionally been seen as a support for knowledge exchange. However, as the COVID-19 pandemic has shown, digital technologies allow new teaching/learning methodologies in digital spaces. Thus, rethinking university spaces is necessary, as places for not only information exchange and production but also meetings and dialogue, in which there is learning by doing.

To achieve this objective, this article first proposes a strategic contribution of conceptual spaces (physical and digital) to integrate smart NZEBs and IoT ecosystems as SDG-enabling technologies. A second executive contribution is an IoT three-level model (data acquisition, interoperable information and knowledge by data-driven decisions) to build a methodology based on continuous cycles of "measure-analyse-decide and act". This iterative methodology allows for efficient adaptation to new building contexts and uses or unexpected circumstances.

\subsection{Conceptual Spaces}

This proposal relates physical space and digital space based on methodologies such as Community [25] and CUA [26], which are related with technological Service Design Thinking models [27]. In physical space, movements and interactions occur with environmental parameters. In digital space, the information obtained by our senses is completed from large amounts of data, acquired in real time by sensors and transformed into information, knowledge and valuable services). As outlined in Figure 2, physical space includes smart sensors (e.g., $\mathrm{CO}_{2}$, humidity, temperature, light, presence and energy consumption) and user experiences (e.g., ventilation, thermal comfort, consumption and cost savings, and energy efficiency). In parallel, digital space includes systems and networks (e.g., interconnection devices, IoT gateways, databases, cloud computing services and visualisation dashboards) to create a digital education ecosystem with customised services, such as on-line classrooms, e-learning tools, virtual teaching technologies and smart campuses, for students, teachers and university staff. The challenge is to act on physical space from digital space. This means to project and install a complete network of sensors and data acquisition devices to monitor, control and visualise space behaviours. The results lead to data-driven decisions, energy savings, energy efficiency and cost-effective solutions, providing knowledge-based value. 


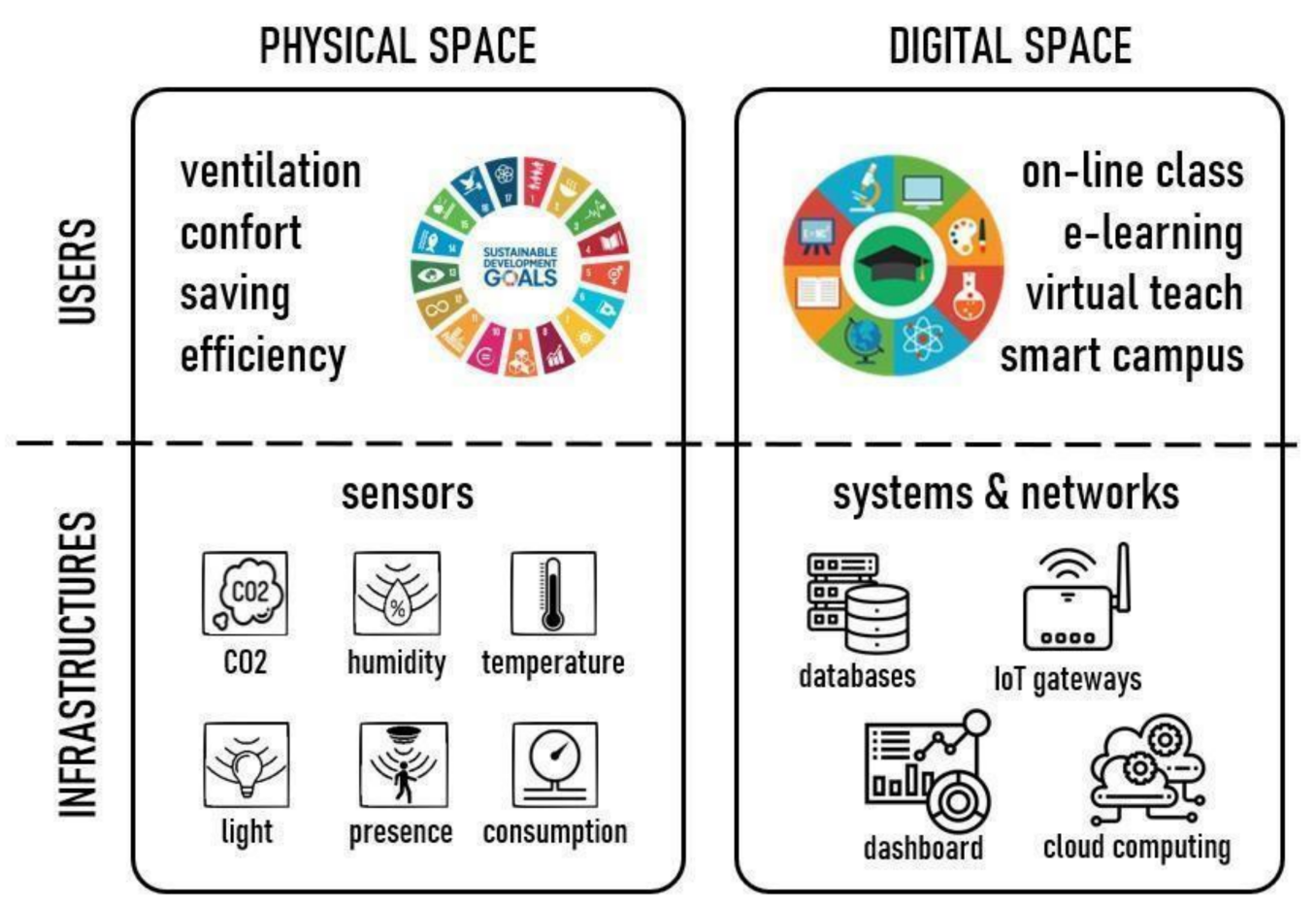

Figure 2. Infrastructures and users as conceptual spaces (physical and digital).

\subsubsection{Physical Space}

In this work, at the infrastructure level, several sensors were included to measure $\mathrm{CO}_{2}$ level (ppm), relative humidity $(\%)$, temperature $\left({ }^{\circ} \mathrm{C}\right)$, light (lumens), presence (pax) and energetic consumption ( $\mathrm{kWh}$ ) (see Figure 2). At the user level (the dimension in which people move and perceive these air conditions, humidity and lighting, thermal comfort, etc.), several of the services include looking for location indications, requesting information about open/closed windows or empty /occupied classrooms and offices and having security in case of emergency, among others. In short, our user experience-at individual and collective levels-within a building is influenced by internal and external conditions, such as lighting or weather, and generates an impact both on cost savings and energy efficiency (see Figure 2). Thus, this evolution of university buildings towards an experimental lab test launches transversal actions, improves the response to society, and reduces the environmental impact while increasing the economic and social impact on the SDGs. With this information and real-time responses, better experiences can be promoted. In addition, the use of spaces can be optimised, improving their habitability, indoor climate and meeting accommodations. Within the university environment is a catalyst for dialogue, activity and the exchange of ideas and knowledge. Smart physical spaces allow tangible interaction with the building with the following value-added services:

- Parameterise, characterise and model each one space, studying its status and behaviour for different uses and external conditions.

- Monitor energy consumption, weather variables, installation performance, etc. to move towards smart buildings.

- Incorporate renewable energies and visualise their utilisation, production, consumption, efficiency, savings, etc.

- Follow up indoor thermal conditions, indoor air quality, occupancy levels, etc.

- Assess and extrapolate the obtained results to added-value SDG-aligned services, such as smart use, energy efficiency and rehabilitation, and predictive and adaptive maintenance.

- Improve DCV, as smart ventilation strategy, based on the patterns obtained from the measured data $\left(\mathrm{CO}_{2}\right.$, humidity, temperature, etc.). 
- Produce renewable (photovoltaic) energy from the smart and digital infrastructures.

- Transform traditional spaces into green and environmentally sustainable spaces for digital education.

- Facilitate the decision-making processes for the smart use of every space.

\subsubsection{Digital Space}

In this work, at the infrastructure level, several systems and networks were included for various purposes, such as air conditioning based on weather and setting lighting levels according to classroom occupation and timetables. This is achieved through IoT gateways, interoperable databases, cloud computing services, visualisation dashboards, etc. (see Figure 2). At the user level (the dimension in which information managed at the infrastructure level evolves into knowledge), several smart services of digital education were included: on-line classrooms, e-learning tools, virtual teaching, smart campuses, etc. (see Figure 2). This digital space can be a teaching/learning environment that integrates full-, semi- and non-face-to-face methodologies, incorporating technological tools, such as e-learning platforms or on-line meeting tools. This flexible adaptation to the specific needs of every user profile (faculties, staff, students and visitors) may allow some of the following features:

- A unified and friendly environment to easily access a virtual and customised classroom, teaching-learning contents, university management, etc.

- Open tools to improve consolidated environments.

- Visibility of class offerings, including their methodologies and transdisciplinary learning, to enable students to choose their own curricula.

- Transparency of the quality teaching offerings, facilitating the integration and access of incoming students regarding their interests, training objectives, etc.

- An active, adaptive and transparent environment for university management, using metrics and quality results.

- Digital twins as virtual support to show available services in a unified and harmonised way to the university community.

- Integration of all digital spaces (classrooms, offices, laboratories, webinars, etc.) to interconnect users (students, teachers and university staff) with academic and management activities.

- Access, in a continuous, adaptive, transparent and user-friendly way, to heterogeneous services classes, practices, jobs, projects, tutorials, evaluation, reservations, notices and messaging, through open and geopositioned data.

\subsection{IoT Three-Level Model}

From previously detailed concepts of physical and digital spaces, this article proposes an IoT three-level model: data-information-knowledge, as outlined in Figure 3. In this context, we considered the definitions for data, information and knowledge. Data consists of raw facts represented by numbers, sounds, images, measurements, etc. Information is the collection of data organised and processed to provide additional value beyond the individual facts. The added value depends on the relationships defined and identified in the dataset. Finally, Knowledge is the understanding of a set of information and the ways that information can be made useful to support specific tasks or make decisions, i.e., to decide and act. 


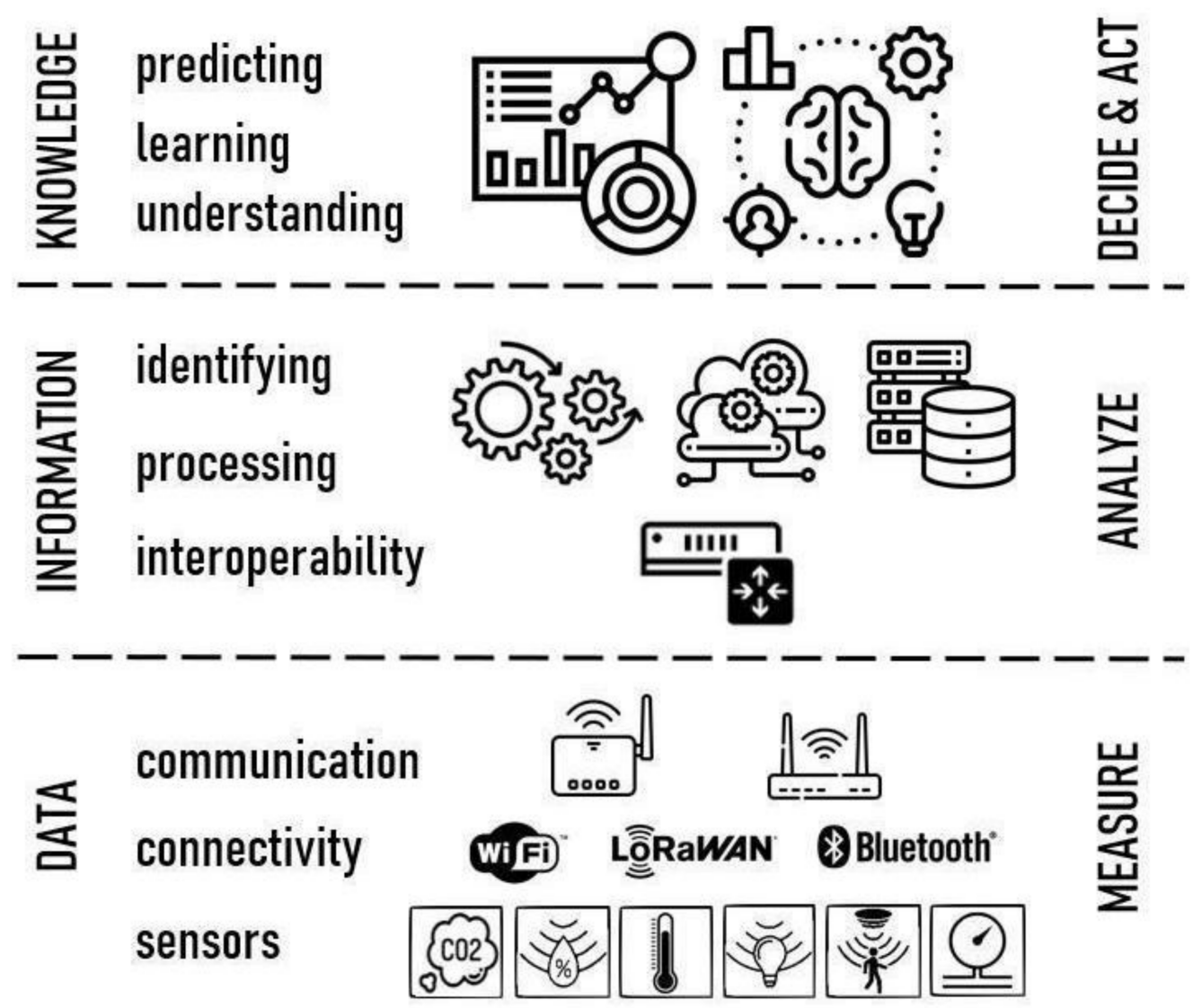

Figure 3. IoT three-level model for "measure-analyse-decide and act" methodology.

\subsubsection{Data Level}

Data are obtained from different kinds of sensors and systems that measure various parameters of interest, such as the following:

- $\mathrm{CO}_{2}$, lighting levels, temperature and humidity in different locations of the university buildings, e.g., corridors, offices, classrooms, restaurants and libraries, to assess thermal comfort and air quality conditions and determine when to use air conditioning systems or natural ventilation (opening windows, ventilation systems, etc.).

- Number/presence of people in the different rooms, obtained by different Quick Response (QR) code verification systems and mobile devices monitoring.

- Real-time electricity consumption, identifying unforeseen consumption, detecting unwanted ignitions, analysing specific information through Supervisory Control and Data Acquisition (SCADA) systems, such as temperatures in the heat-transfer fluids in supply and return.

- Electricity generation levels of the photovoltaic panels in order to analyse the efficiency of the renewable energies.

- Timetables and other parameters related to the mobility of the people in the university buildings, such as heat maps and access frequencies.

As shown in Figure 3, these collected data are sent to the following communication levels by various interconnected devices through their associated connectivity technologies, such as Bluetooth, WiFi and LoRaWAN, among others. In more detail, in the Section 3.1, a six-layer ecosystem based on this IoT three-level model, developed as a proof-of-concept of the methodology proposed in this paper, is also described. Acquisition and Ingestion layers of this six-layer system corresponds to the data level. Thus, Acquisition layer is composed of a network of different kinds of sensors and routers whose main purpose is obtaining data and measuring the different parameters described above. 
Furthermore, the entire process of installation and calibration of sensors and infrastructures were analysed in detail. For example, to guarantee the accuracy of $\mathrm{CO}_{2}$ sensors, the calibration protocol included two criteria. First, from the technical information provided by device manufacturers, a device uncertainty value was determined to be $\pm 50 \mathrm{ppm}$. Second, the dispersion of the measurement variation was studied through a reference device (testo480@) with a calibration certificate [28]. The revision thresholds were then established as $+65 \mathrm{ppm}$ (upper limit) and $-55 \mathrm{ppm}$ (lower limit) with respect to the reference device. Figure 4 shows the dispersion of the measurement variation for several sensors (indicated by their location codes (floor.space as $0.01,0.02$, etc.). Thus, those sensors that were not between the calibration thresholds $(0.01,0.04$ and 3.09) required calibration. Furthermore, all collected data are sent to the Information level (see Section 2.2.2) through the Ingestion layer (see Section 3.1), which is composed by a set of gateways and streaming techniques to enable the subsequent data processing.

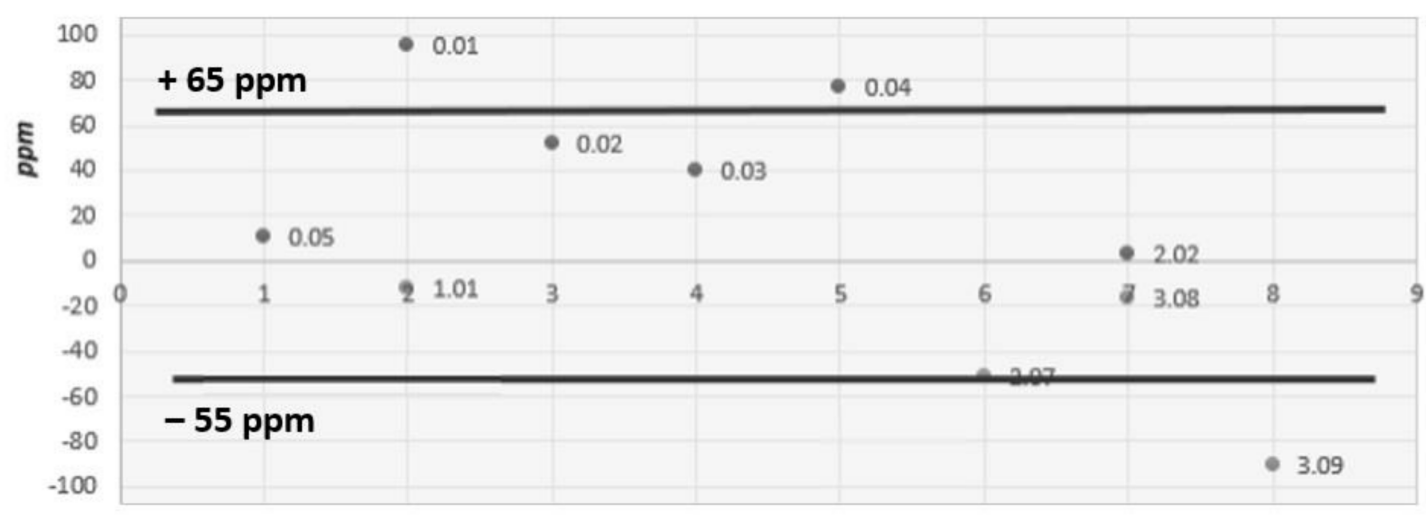

Figure 4. Calibration thresholds respect the certified reference device for $\mathrm{CO}_{2}$ measurement.

\subsubsection{Information Level}

These sensors and systems typically do not save measured data, but they are connected to various networks in which nodes receive and process $=$ transform the data into information. These networks use different protocols to transmit data among nodes, such as a Programmable Logic Controller (PLC), depending on their features (consumption, cost, etc.). Moreover, network protocols used by these devices are not normally based on Transmission Control Protocol and Internet Protocol (TCP/IP) standards, which are generally used to optimise and manage the storage and retrieval of data and information. Thus, sensor networks end up in a concentrating element (e.g., SCADA system or Lora Server node) that acts as an IoT gateway to send the data to a TCP/IP network achieving the interoperability of the communication (see Figure 3). In the proposed six-layer ecosystem (see Section 3.1), the Processing and Storage layers correspond to this level.

In more detail, different processes based on Extraction, Transformation and Load (ETL) rules are applied to save and store the organised data by considering different relationships relevant for the domain considered. In addition, data are associated with metadata according to the best practices and standards defined by FAIRMODE, W3C, OGC-INSPIRE and other relevant specifications for the domain considered. Moreover, before proceeding to the next level, all information is analysed by iterative processing for consistency and reliability. Suitable data with a certain level of quality and appropriate data organisation are key to the generation of knowledge, avoiding bias or poor conclusions, because missing, incorrect or invalid data can compromise the result [12]. Thus, statistical techniques to identify outliers and missing data are applied to verify the quality of the data and adjust, if necessary. 


\subsubsection{Knowledge Level}

The general purpose of this level is the development of services to predict, analyse and visualise the use and performance of non-residential buildings by considering different models and parameters (see Analysis and Visualization layers in the Section 3.1). These services generally make use of High-Performance Computing (HPC) technologies when a large set of heterogeneous and historical data is considered. In particular, three types of services are proposed:

- Services to detect inadequate operations in the facilities, such as malfunctioning systems, equipment that should be turned off at certain time slots, lower than expected performance, and high energy consumption.

- Predictive services with multiple applications, such as those able to predict the real performance of the facilities under specific circumstances and comfort parameters for users. For this purpose, learning how the non-residential buildings work in real scenarios is required. Thus, monitoring and acquiring historical data of the buildings and their context is key, e.g., data concerning the energy consumption of heaters and pumps, thermal inertias, and parameters about the passive behaviour of different rooms and buildings.

- Prescriptive or recommendation services that support suitable data-driven decisionmaking. For example, knowledge about the behaviour of Heating, Ventilating and Air Conditioning (HVAC) can help determine the timetable to switch the facilities and equipment of the buildings on/off. As another example, Model Predictive Control (MPC) supports an automatic and efficient control of the facilities (under a series of registry inputs) by considering the predictions obtained with the models.

To develop these services, different types of models are considered: physical (whitebox) models, models based only on data (black-box) and hybrid (grey-box) models [29]. Hybrid models are often considered the most effective at balancing the trade-off between computational costs and prediction accuracy. A crucial task for the success of these services is the verification and validation/analysis of the considered models to determine the quality of the results.

\section{Results}

Following the "measure-analyse-decide and act" methodology, an IoT ecosystem (named sensoriZAR) was implemented as a proof-of-concept of a smart campus at the University of Zaragoza, Spain. This smart campus includes three buildings: Ada Byron (built in 1998 with 14,000 $\mathrm{m}^{2}$ for 1000 students and 250 university staff), Torres Quevedo (built in 1986 with 21,000 $\mathrm{m}^{2}$ for 1500 students and 350 university staff) and Agustin de Betancourt (built in 1999 with $27,000 \mathrm{~m}^{2}$ for 2000 students and 450 university staff). This work details the obtained results, focused on $\mathrm{CO}_{2}$ and energy consumption monitoring, during the recent design, development, implementation and technical validation of sensoriZAR.

\subsection{Architecture}

Firstly, the architecture of the sensoriZAR IoT ecosystem is presented, which includes both the infrastructure and the technological tools to create and manage all the processes within the "measure-analyse-decide and act" methodology. This architecture was designed to store a large volume of heterogeneous data (acquired at high speed), transform it into information, and provide added-value knowledge. The architecture is made up of six layers (see Figure 5): acquisition, ingestion, processing, storage, analysis and visualisation. 


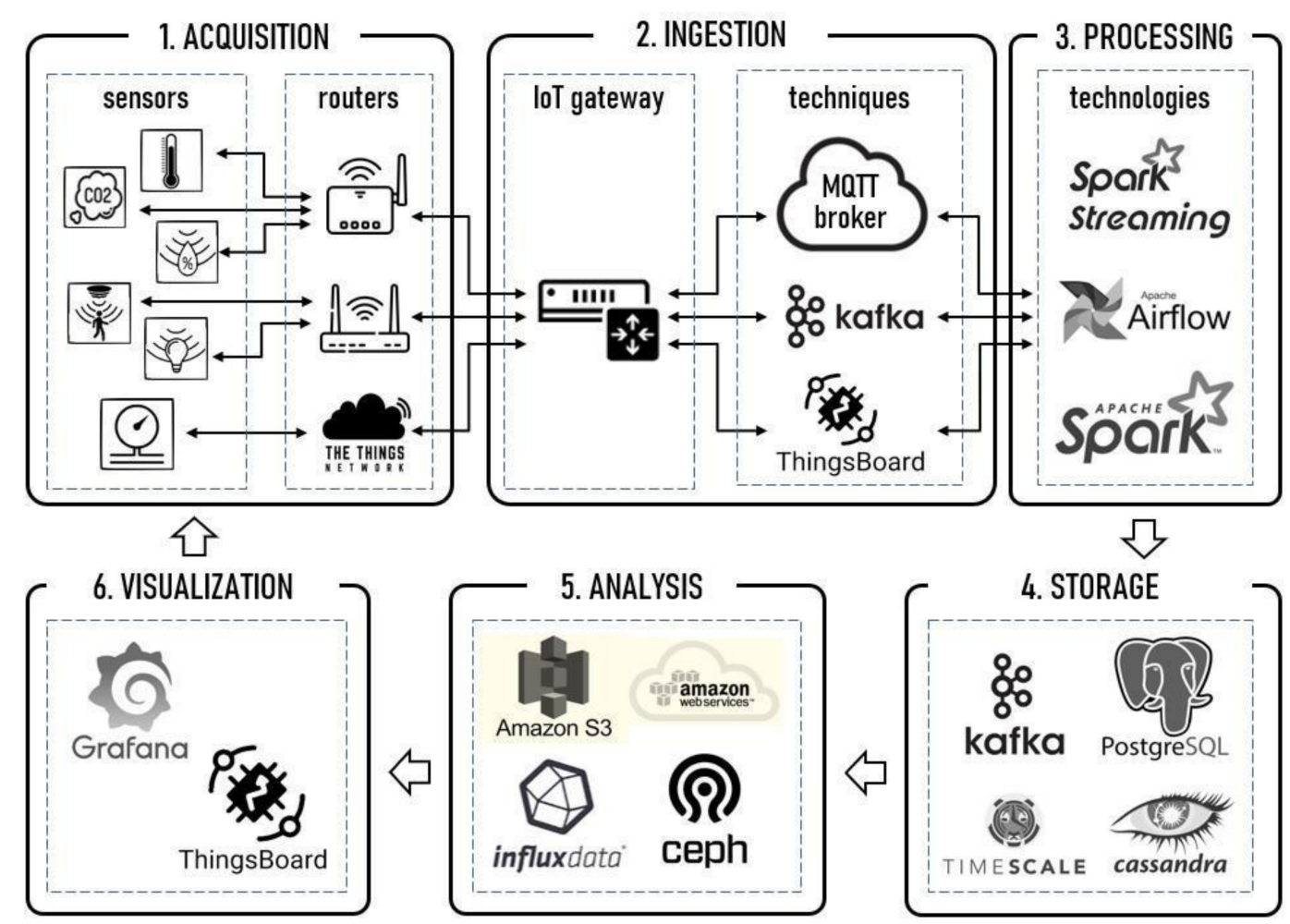

Figure 5. Architecture and functional diagram of sensoriZAR.

- Acquisition. The first step is the origin of the data: where it is generated, at what speed, with what quality, etc. Firstly, the acquisition layer solves the heterogeneity of sensors, brands, models, manufacturers, etc. Secondly, this layer harmonises the connectivity problem between heterogeneous technologies, protocols, interconnection devices, etc. that will communicate in an interoperable way with the IoT gateway at the next layer.

- Ingestion. The ingestion layer is responsible for the data through the IoT gateway. It can include a wide variety of techniques, such as publication, subscription, batch processes and continuous flows. For this work, the proposed ingestion layer consisted of an IoT messaging server (MQTT broker), an event platform (Kafka) and a device management application (ThingsBoard). Thus, ingestion works through components specifically created for data collection with non-native sources or with indirect or restricted access.

- Processing. After the ingestion layer, the processing layer allows data transforming, if necessary, for later storage. Data processing can be implemented by batch or flow processing. Multiple technologies can perform this task, each with a specific purpose. Some technologies used in this project (all open-sourced) were Spark Streaming (for real-time, scalable data processing), Airflow (to manage, monitor and plan workflows) and Spark (as a clustered computing framework for distributed data processing).

- Storage. Generated data needs a medium storage, for subsequent consultation and analysis. To handle a large dataset, distributed storage systems, object repositories or specific storage technologies, such as graph and time-series databases, are required. Because of the data heterogeneity, this work used various storage technologies, including Kafka (as a distributed storage for raw data), PostgreSQL (as an objectoriented relational database management system), TimeScaleDB (as an optimised database for storing data as time series) and Cassandra (as a distributed storage, specific for time series). Thanks to this versatility, a broad integration of systems and services was achieved, along with the scalability, availability and reliability of the entire IoT ecosystem. 
- Analysis. Many technologies exist for the extraction of characteristics, analysis and experimentation with the stored data. Because the tools are open-source, using standard formats and protocols, the architecture is flexible enough to apply different technologies depending on the analysis objective. This project, specifically for the heterogeneous features of the collected data, combined several big data analysis technologies, including Amazon Web Services (as tools and services set for cloud computing), Amazon S3 (as a specific service for secure objects management in the cloud), Ceph (as an open analysis system, defined by software, specific for large amounts of distributed data) and influxdb (as a reliable management system to visualise data time series, specific for IoT), among others.

- Visualisation. Finally, the visualisation layer is compatible with various display technologies, panels, browsers, etc. For this work, two environments were integrated: ThingsBoard (for short-term monitoring and control) and Grafana (for structured information from different data sources).

\subsection{Infrastructures and Users}

Secondly, following the conceptual spaces proposal, this architecture was implemented at infrastructure and user levels, detailing in both cases their physical and digital spaces.

At the infrastructure level, Table 1 describes several alternatives to set-up implementation by electing the best suitability and minimal cost. Firstly, wired solutions were discarded because of their installation problems and combinations with wired sensors. Wireless gateways were also discarded due to their connectivity problems. Following this, wireless sensors were analysed with both commercial solutions and open IoT cloud networks. This last option was chosen for its good scalability and high adaptability with a medium cost. The wireless technology chosen was LoRaWAN because of its excellent indoor range, ubiquitous connectivity and high technological adoption [30].

Table 1. Costs and range comparative for set-up election.

\begin{tabular}{cccccc}
\hline Set-Up & $\begin{array}{c}\text { Sensor } \\
\text { Cost }(\boldsymbol{\ell})\end{array}$ & $\begin{array}{c}\text { Connectivity } \\
\text { Cost }(\boldsymbol{\epsilon})\end{array}$ & $\begin{array}{c}\text { Indoor } \\
\text { Range }(\mathbf{m})\end{array}$ & Disadvantages & Advantages \\
\hline $\begin{array}{c}\text { wired sensor } \\
\text { wired gateway }\end{array}$ & $10-25$ & $150-250$ & $100-250$ & installation & $\begin{array}{c}\text { low cost } \\
\text { reliability }\end{array}$ \\
\hline $\begin{array}{c}\text { wired sensor } \\
\text { wireless gateway }\end{array}$ & $10-25$ & $150-250$ & $15-40$ & connectivity & $\begin{array}{c}\text { low cost } \\
\text { high adaptability }\end{array}$ \\
\hline $\begin{array}{c}\text { LoRaWAN sensor } \\
\text { commercial solutions }\end{array}$ & $250-400$ & $450-900$ & $1000-1500$ & $\begin{array}{c}\text { high cost } \\
\text { low adaptability }\end{array}$ & good scalability \\
\hline $\begin{array}{c}\text { LoRaWAN sensor } \\
\text { open IoT network }\end{array}$ & $250-400$ & $250-400$ & $1000-1500$ & medium cost & $\begin{array}{c}\text { good scalability } \\
\text { high adaptability }\end{array}$ \\
\hline
\end{tabular}

With these premises, meaningful variables were real-time monitored from wired and wireless sensors. Wired sensors (over 300) were connected to the SCADAs to provide the airquality parameters detailed in [31]. Wireless LoRAWAN sensors (46) were ad-hoc installed to provide temperature, humidity, barometric pressure and $\mathrm{CO} 2$ level. The selected sensors were SenseCAP AU915 [32] and Aranet 4 Pro [33]. Their features, technical specifications and device details are summarized in [32] and [33], respectively. Figure 6 shows several examples of their real installation in the three buildings of sensoriZAR: 15 in Ada Byron, 12 in Torres Quevedo, 19 in Agustín de Betancourt. 


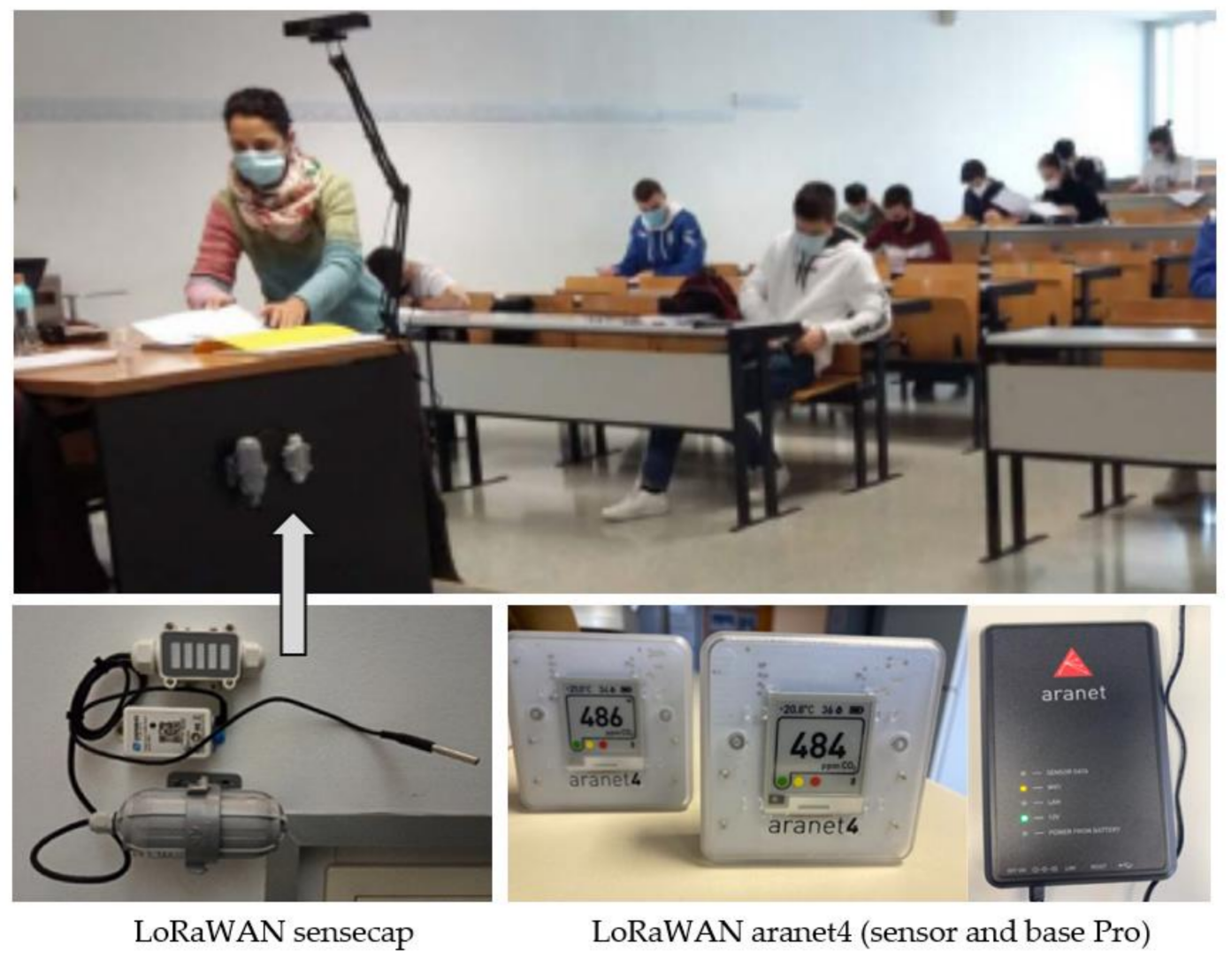

Figure 6. Sensecap [32] and Aranet [33] LoRaWAN sensors and their real installation in sensoriZAR.

At the user level, several user profiles were considered with different roles and interactions with sensoriZAR:

- Directorate and research-responsible for long term vision, coordination and system design.

- IT and IoT units-responsible for computer networks, servers and IoT infrastructure implementation.

- Technical, security, green office units-responsible for building subsystems (lighting, HVAC, alarms, etc.)

- Maintenance and concierge-responsible for daily operation, data monitoring and problem solving.

- Students and university staff-main users of the system as data consumers and generators.

Each user profile requires different outputs from the system. For example, IT and IoT units need to monitor infrastructure to ensure the correct functioning of the system. Technical units are the main data producers that aim to consume big data generated from other units. Maintenance and concierges are notified when an issue needs to be addressed (e.g., $\mathrm{CO}_{2}$ level above $800 \mathrm{ppm}$ or a malfunctioning sensor). Finally, students and staff are interested in their closest environments (or where they are heading), e.g., the current $\mathrm{CO}_{2}$ level in the room.

In order to satisfy all needs, the user interface is able to provide a visual one-glance and colour-based perspective of how all the spaces in the buildings are working (see Figure 7). The left area of Figure 7 shows all the sensors installed in the smart campus, grouped by building and ordered by floors. A green circle means good performance with a $\mathrm{CO}_{2}$ level between 390 and 700 ppm; yellow means necessary ventilation with a $\mathrm{CO}_{2}$ level between 700 and 850 ppm; red means a non-secure area with a $\mathrm{CO}_{2}$ level higher than 850 ppm; the tool icon means a necessary revision with $\mathrm{CO}_{2}$ level lower than $390 \mathrm{ppm}$. The right area of Figure 7 shows in detail every real-time measurement from the selected sensor 
and the $\mathrm{CO}_{2}$ level (ppm), temperature $\left({ }^{\circ} \mathrm{C}\right)$, relative humidity $(\%)$ and presence (pax). Furthermore, different mechanisms can be used to download data for third-party use and data-driven decisions. This hierarchical navigation for each building's space with detailed visualisation (all variables and short-term data) was useful as indicated by users in the first user-experience validations.

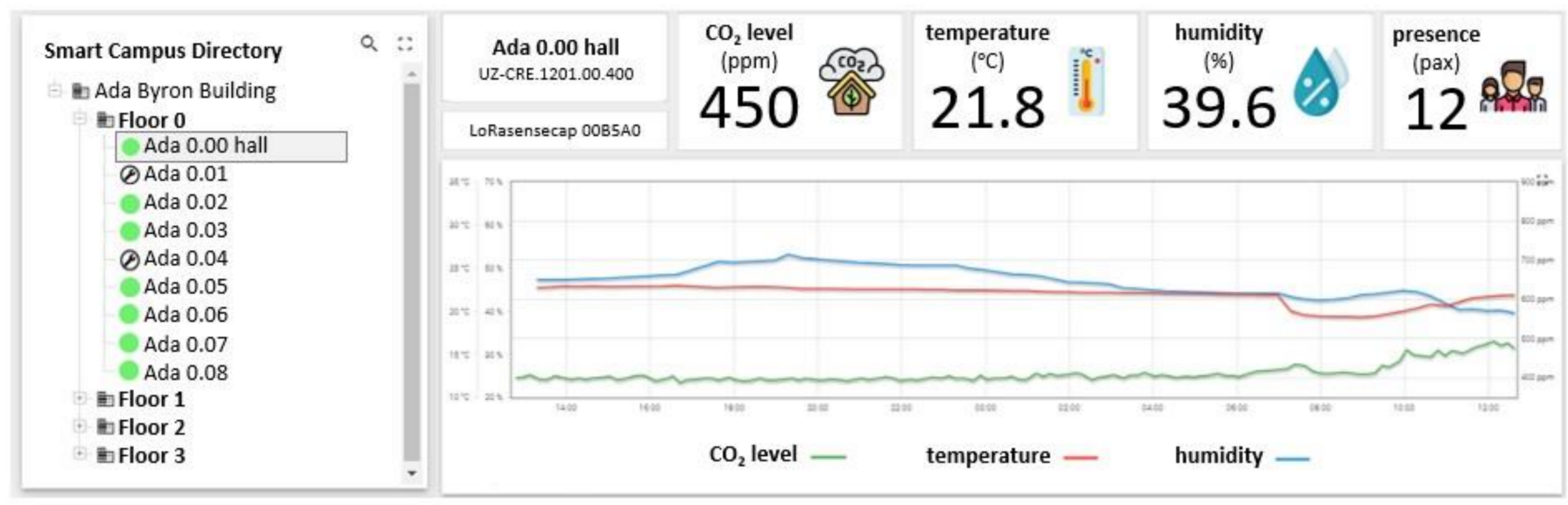

Figure 7. Dashboard for real-time visualisation and data-driven decisions.

\subsection{Success Stories as a Proof-of-Concept of a Smart Campus}

The aim of this work serves as both a proof-of-concept of a smart campus and an application model in academic works, research projects and institutional initiatives; several success stories are presented. The first examples are focused on energy consumption monitoring and, derived from the COVID-19 situation, $\mathrm{CO}_{2}$ evolution as analysed in a specific classroom.

On the one hand, to quantify the instantaneous and seasonal value of the campus performance, the main indicators of the heat pumps were analysed: Energy Efficiency Ratio (EER), Coefficient Of Performance (COP), Seasonal EER (SEER), and Seasonal COP (SCOP). The buildings operate with an open-cycle geothermal heat pump system, using groundwater ( $30 \mathrm{~m}$ deep). As shown in Figure 8 (with results obtained from data analysis from the last three years), the groundwater temperature is stable, although changes according to the season. Furthermore, from the SCADA analysis, EER is roughly 6, a value useful as a real-time indicator. Thus, EER evolution shows the influence of maintenance operations or variations in boundary conditions (refrigerant change, exchangers cleaning, compressor repair, machine parameters programming, etc.). This indicator contributes to improving the SRI of the building in the heating and cooling system.

These studies have also allowed measurements of Energy Performance Indicators (EnPIs), as ISO 50001:2018 [34] recommends, such as the above-mentioned SEER and SCOP and Energy Baselines (EnBs). These indicators serve as quantitative tools to annually audit the installation and track both the device deterioration and the improvements implemented each year. As shown in Figure 9 (detailing EnBs for one of the campus building from 2015 to 2018), the consumption baseline holds a consistent behaviour. Thanks to the proposed methodology of persistent data collection and analysis, inefficiencies in the operation of hydraulic water pumps were detected from the groundwater to plate exchangers-the pumps operated for more hours than necessary for the air conditioning service with a significant electrical overrun (which was later reduced with remarkable savings). 


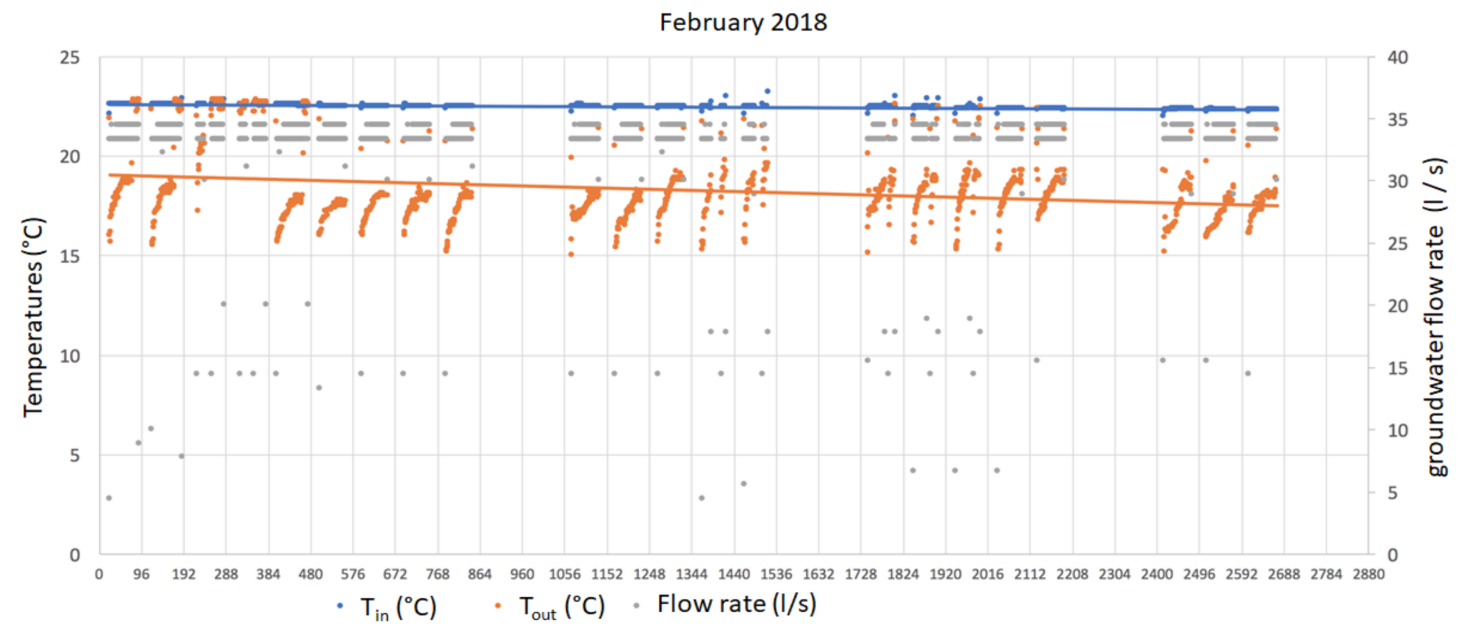

Figure 8. Temperature (taken and returned to the ground) and flow rate measured in the groundwater used by the heat pump.

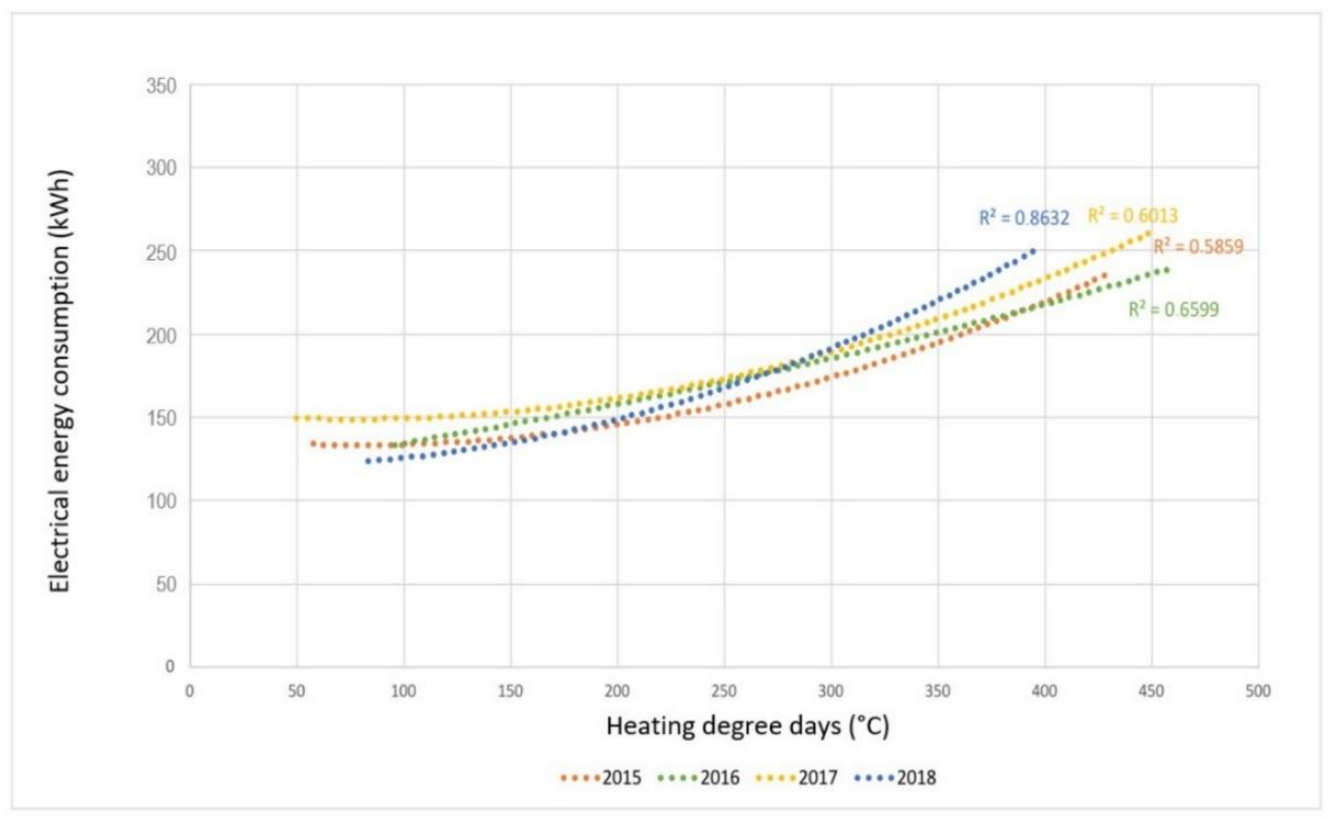

Figure 9. Energy Baseline (EnBs) of Betancourt building (2015-2018).

Another key point of efficiency improvement through the proposed methodology was the smart management that kept the secondary circuit pumps running longer (after the primary circuit stop) to continuously harness thermal energy from the heat-transfer fluid in the building.

On the other hand, the "measure-analyse-decide and act" methodology applied to spaces has shown the significance of situations of energy inefficiency. Some examples include the effective occupation of university spaces, detection of unnecessarily airconditioned areas outside working hours and unnecessary illumination of empty areas, among others. The use of intelligent systems allows the detection in real time of inefficiencies that condition the operation of buildings, and that taking measures allows making their management more efficient. Figure 10 shows a comparative analysis of the energy consumption (electricity in blue and gas in red) of the different university buildings. As Figure 10 illustrates, the diversity of construction and facilities of the university's building is very wide. Until the implementation of intelligent monitoring systems, the consumption data of the buildings were obtained from the energy billing and the information provided from the 
general electrical totalizers of the buildings. Current real-time monitoring is automatically integrated with SCADA through Siemens Sentron Pac 3200 digital energy multimeter [35]. These consumptions have even been compared to standards levels: $238 \mathrm{kWh} / \mathrm{m}^{2}$.year (following Technical Building Code, CTE [36], as the green threshold) and $120 \mathrm{kWh} / \mathrm{m}^{2}$.year (following Passivhaus international recommendation, as the orange threshold). As shown in Figure 10, the three buildings of the proposed proof-of-concept of a smart campus (Torres Quevedo, Agustin de Betancourt and Ada Byron in black) fulfil the CTE standard. These comparative studies allow the detection of both behavioral anomalies and a correct performance. Even so, they must be completed with intelligent monitoring systems such as those carried out with the aim of being able to deepen the inefficiencies and in the possible solutions [37].

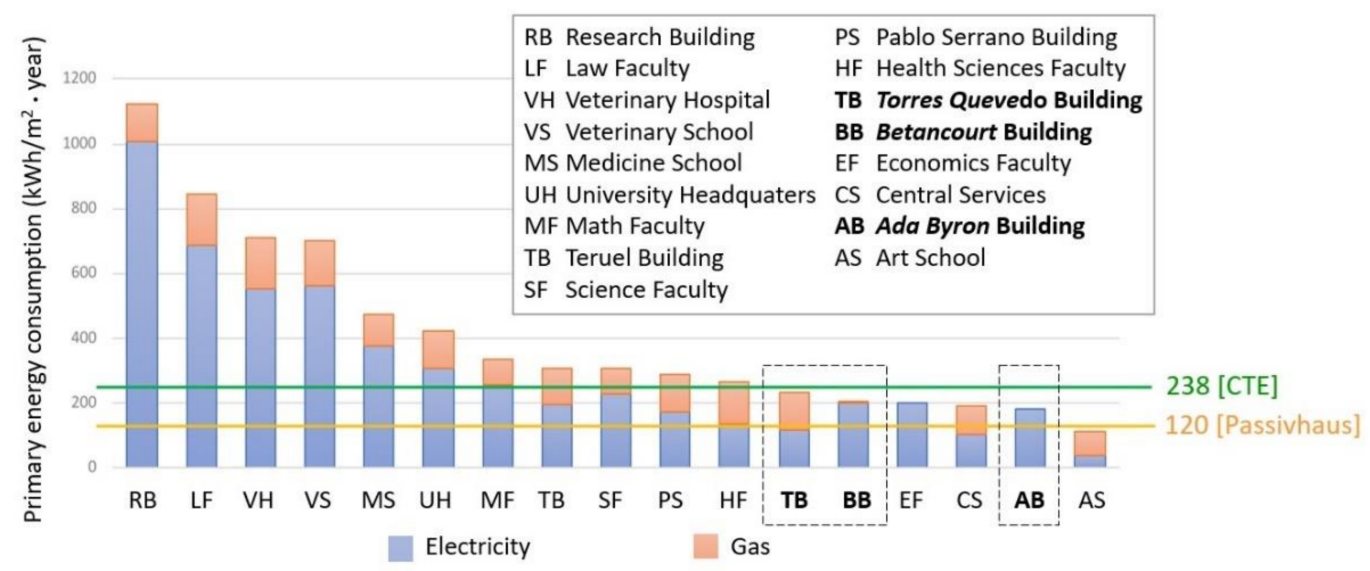

Figure 10. Primary energy consumption $\left(\mathrm{kWh} / \mathrm{m}^{2}\right.$.year) for different buildings, indicating the smart campus fulfils the standard threshold.

Finally, and as an example of the importance of facility analysis thanks to monitoring and follow-up, all this analysis showed the improvements obtained as detailed in Figure 11 in terms of economic cost and energy consumption. Figure 11 shows a reduction of more than $50 \%$ when evolving from buildings that use gas boiler (for heating and cooling) with compression chillers with outdoor air focus $\left(20.9-27.4 € / \mathrm{m}^{2}\right.$ and $\left.414 \mathrm{kWh} / \mathrm{m}^{2}\right)$ to HVAC buildings with heat pumps using groundwater $\left(10-14 € / \mathrm{m}^{2}\right.$ and $\left.194 \mathrm{kWh} / \mathrm{m}^{2}\right)$.

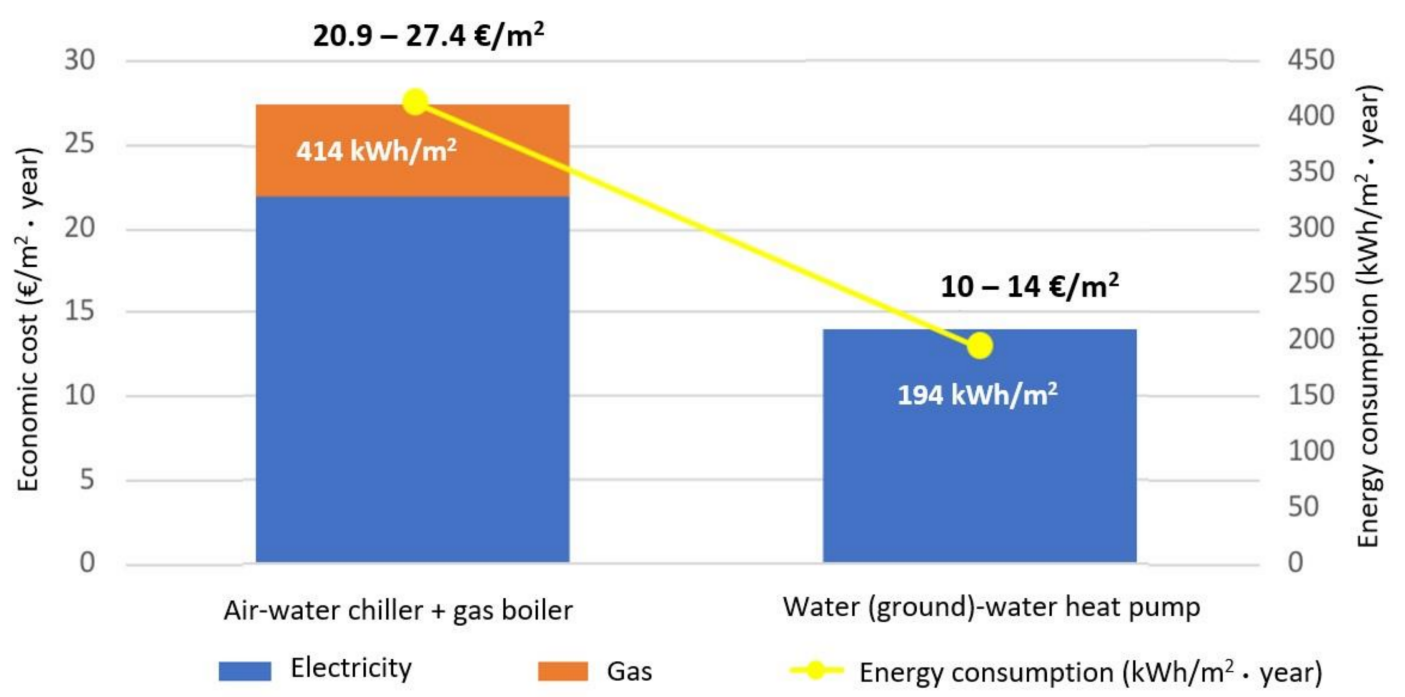

Figure 11. Economic cost $\left(€ / \mathrm{m}^{2}\right.$.year $)$ and energy consumption $\left(\mathrm{kWh} / \mathrm{m}^{2} \cdot\right.$ year $)$ in university buildings: improvement obtained in the smart campus. 
Derived from the COVID-19 situation, the system proved its utmost relevance to tackle high-risk situations. Figure 12 shows the $\mathrm{CO}_{2}$ evolution in a specific classroom and the effectiveness of the system when the value approached the $700 \mathrm{ppm}$ level but stayed below the 800 ppm threshold. This generated invaluable trust of the institution, not only of the students but also of the staff.

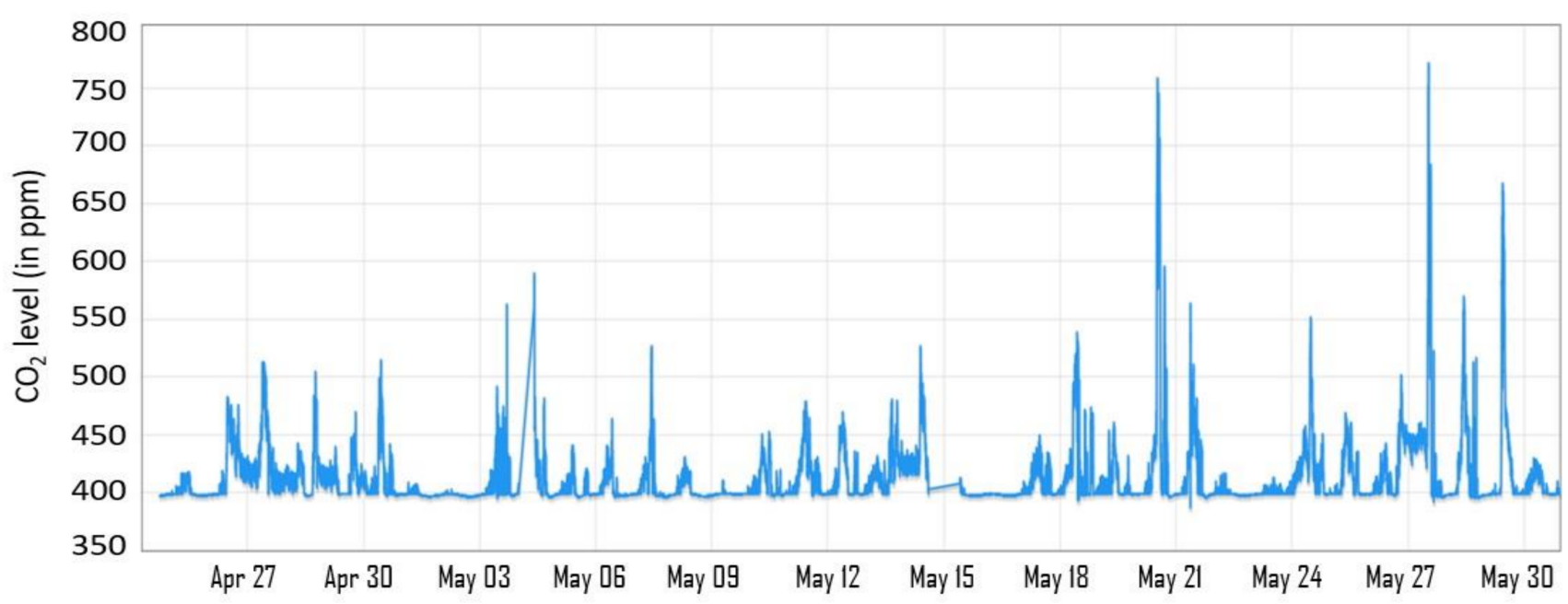

Figure 12. $\mathrm{CO}_{2}$ evolution in a specific classroom.

\section{Discussion}

The results of the proof-of-concept of a smart campus demonstrate that quantitative measurements are key elements for subsequent analyses and decision-making processes. The information collected through real-time monitoring of university buildings showed both the data utility for continuous improvement and the need to increase the number of measurement devices to cover all campus spaces. Furthermore, the proposed approach is committed to open science in order to foster verifiable scientific knowledge subject to scrutiny and critique, the reproducibility of the experimental results, and robust decisionmaking processes.

Measurements of $\mathrm{CO}_{2}$, temperature and humidity (among other key parameters) in every space work toward an objective and well-founded approach to establishing air conditioning schedules (analysing the inertia of the building) and HVAC improvements. Thus, the proposed methodology assesses the performance of the installation and generates quantitative indicators of the operation system, following the SRI perspective that the EPBD recommends [21]. Having quantitative patterns of HVAC behaviour will allow for the adjustment of the ventilation systems. For example, opening the windows (losing thermal comfort) in those spaces can be avoided with a correct ventilation air supply through HVAC ducts and diffusers. Furthermore, the digitisation of the entire ecosystem will allow data-driven decisions to move towards the automation of DCV systems.

Likewise, to obtain higher energy efficiency, both the main building energy consumption and performance HVAC indicators (COP and EER) should be monitored. Additional ventilation control, such as the better use of free-cooling hours (night and early morning) with outdoor air in summer, along with local demand control based on air-quality sensors $\left(\mathrm{CO}_{2}\right)$ with damper-regulated local flow regulated (level 4, maximum level, SRI ventilation), can optimise ventilation consumption.

SRI of some university buildings has begun to be evaluated to contribute to transversal knowledge of smart campus. The first evaluated building has obtained a value of SRI $=16.45$ (over 100). The use of this methodology has provided a large number of ideas for improvement in terms of monitoring, regulation and control, to improve the capacity of the building to adapt to the demands. 
These lessons learned lead us to continue taking steps towards digital twins. Experiments are being carried out to obtain predictive models to characterise spaces using solid $\mathrm{CO}_{2}$ pellets and analysing the evolution of $\mathrm{CO}_{2}$ reduction curves in the classroom. These models are valuable for users with new (collaborative and interactive through digital technologies) teaching/learning methodologies. In addition, the models help infrastructures with smart automation, regulation and control, becoming more efficient, effective, flexible and sustainable.

\section{Conclusions}

Post-pandemic challenges to fulfil SDGs and improve energy efficiency require holistic contributions and data-based methodologies as it is proposed in this work. The need to measure to turn data into knowledge and quantify key efficiency indicators, such as SRI, is essential to make data-driven decisions. This paper contributed an open data IoT ecosystem (sensoriZAR), defining two conceptual spaces (physical and digital) based on Service Design Thinking, within two dimensions (for users and for infrastructures) over an IoT three-level model (data, information and knowledge). As a proof-of-concept of IoT and SDG-enabling technologies towards NZEB, the results showed that evolving to a smart campus as experimental lab tests can achieve energy efficiency, performance audits, ultra-low consumption, and cost savings. Furthermore, this proposal of a university reference model (for academic works, researching projects, institutional initiatives, etc.) is extendable to smart buildings and cities and compatible with complementary proposals from other universities and initiatives to establish transversal contacts (through articles like this one) for joint collaborations.

Author Contributions: This research group is multidisciplinary and all the authors have contributed in the entire manuscript. All authors have read and agreed to the published version of the manuscript.

Funding: This research received no external funding.

Data Availability Statement: All the published data can be found in sensoriZAR ecosystem (https: / / sensorizar.unizar.es / (accessed on 7 July 2021).), Green University Office (https: / / oficinaverde. unizar.es/ (accessed on 7 July 2021).) and Open Data and Transparency Portal University of Zaragoza (https:/ / datuz.unizar.es/ (accessed on 7 July 2021).).

Acknowledgments: Authors thanks to Engineering and Architecture School (EINA), Green University Office and University of Zaragoza for their support in this work.

Conflicts of Interest: The authors declare no conflict of interest.

\section{References}

1. European Court of Auditors (ECA). Special Report. Energy Efficiency in Buildings: More Emphasis on Profitability. 2020. Available online: https:/ / www.eca.europa.eu/Lists /ECADocuments/SR20_11/SR_Energy_efficiency_in_buildings_ES.pdf (accessed on 7 July 2021).

2. EU Council. Executive Conclusions. Building a Sustainable Europe by 2030. Progress Thus Far and Next Steps. 2019. Available online: https:/ / www.consilium.europa.eu/media/41693/se-st14835-en19.pdf (accessed on 7 July 2021).

3. European Commission. A Renovation Wave for Europe. 2020. Available online: https://ec.europa.eu/energy/topics/energyefficiency / energy-efficient-buildings/renovation-wave_en (accessed on 7 July 2021).

4. European Commission. A European Green Deal. Striving to Be the First Climate-Neutral Continent. 2020. Available online: https:/ / ec.europa.eu/info/strategy/priorities-2019-2024/european-green-deal_en (accessed on 7 July 2021).

5. European Union Law. European Climate Law. Proposal for a Regulation of the European Parliament and of the Council Establishing the Framework for Achieving Climate Neutrality and Amending Regulation. 2018. Available online: https: / / eur-lex.europa.eu/legal-content/EN/TXT/ ?qid=1588581905912\&uri=CELEX:52020PC0080 (accessed on 7 July 2021).

6. Assaf, L. Audit procedures for the evaluation of lighting installations in non-residential buildings. In Efficient Lighting Initiative (ELI) Global Environmental Facility-IFC World Bank, EDESUR, National University of Tucumán. 2011. Chapter 16, 35p. Available online: https:/ / www.researchgate.net/publication/317084293_Procedimiento_de_Auditorias_para_la_Evaluacion_ de_Instalaciones_de_Iluminacion_de_Edificios_no_Residenciales (accessed on 7 July 2021).

7. Spanish Government. Long-Term Strategy for Energy Rehabilitation in Building Sector (ERESEE). 2020. Available online: https: / / www.mitma.gob.es/el-ministerio/planes-estrategicos/estrategia-a-largo-plazo-para-la-rehabilitacion-energetica-enel-sector-de-la-edificacion-en-espana (accessed on 7 July 2021). 
8. Dave, E. Internet of Things, How the Next Evolution of the Internet Is Changing Everything. Cisco Internet Business Solutions Group (IBSG). 2011. Available online: https://www.cisco.com/c/dam/en_us/about/ac79/docs/innov/IoT_IBSG_0411FINAL.pdf (accessed on 7 July 2021).

9. Atzori, L.; Iera, A.; Morabito, G. The Internet of Things: A survey. Comput. Netw. 2010, 54, 2787-2805. [CrossRef]

10. Says, G. 6.4 Billion Connected Things Will Be in Use in 2016, up 30 Percent from 2015. Gartner Inc. 2015. Available online: http:/ / www.gartner.com/newsroom/id/3165317 (accessed on 7 July 2021).

11. Ashraf, Q.M.; Yusoff, M.I.M.; Azman, A.A.; Nor, N.M.; Fuzi, N.A.A.; Saharedan, M.S.; Omar, N.A. Energy monitoring prototype for IoT. In Proceedings of the IEEE World Forum on Internet of Things, Milan, Italy, 14-16 December 2015; pp. 1-5, ISBN 978-1-5090-0366-2.

12. Schelter, S.; Lange, D.; Schmidt, P.; Celikel, M.; Biessmann, F.; Grafberger, A. Automating Large-Scale Data Quality Verification. VLDB Endow. 2018, 11, 1781-1794. [CrossRef]

13. UNESCO. Recommendation on Open Science. 2021. Available online: https://en.unesco.org/science-sustainable-future/openscience/recommendation (accessed on 7 July 2021).

14. Durier, F.; Carrié, R.; Sherman, M. Whats Is Smart Ventilation? Air Infiltration and Ventilation Centre, AIVC VIP 38.2018 Available online: https:/ / www.aivc.org/resource/vip-38-what-smart-ventilation (accessed on 7 July 2021).

15. Pantazaras, A.; Lee, S.E.; Santamouris, M.; Yang, J. Predicting the CO2 levels in buildings using deterministic and identified models. Energy Build. 2016, 127, 774-785. [CrossRef]

16. Spanish Government. Report on Prospective and Future Evolution of Air Conditioning and ACS Systems in Tertiary Buildings. 2019. Available online: https:/ / www.mitma.gob.es/el-ministerio/planes-estrategicos/estrategia-a-largo-plazo-para-larehabilitacion-energetica-en-el-sector-de-la-edificacion-en-espana (accessed on 7 July 2021).

17. Tang, S.; Shelden, D.R.; Eastman, C.M.; Pishdad-Bozorgi, P.; Gao, X. A review of building information modeling (BIM) and the Internet of Things (IoT) devices integration: Present status and future trends. Autom. Constr. 2019, 101, 127-139. [CrossRef]

18. Lawal, K.; Rafsanjani, H.N. Trends, benefits, risks, and challenges of IoT implementation in residential and commercial buildings. Energy Built Environ. 2021, in press. [CrossRef]

19. Pocero, L.; Amaxilatis, D.; Mylonas, G.; Chatzigiannakis, I. Open source IoT meter devices for smart and energy-efficient school buildings. HardwareX 2017, 1, 54-67. [CrossRef]

20. Poongothai, M.; Subramanian, P.M.; Rajeswari, A. Design and Implementation of IoT Based Smart Laboratory. In Proceedings of the International Conference on Industrial Engineering and Applications (ICIEA), Singapore, 26-28 April 2018 ; pp. 169-173. [CrossRef]

21. European Commission. Energy Performance of Buildings Directive. 2018. Available online: https://ec.europa.eu/energy/topics/ energy-efficiency/energy-efficient-buildings/energy-performance-buildings-directive_en (accessed on 7 July 2021).

22. European Commission. Smart Readiness Indicator (SRI) for Buildings. 2018. Available online: https://smartreadinessindicator. eu/ (accessed on 7 July 2021).

23. European Commission. Outcomes of the First Technical Study about SRI. 2018. Available online: https://smartreadinessindicator. eu/1st-technical-study-outcome (accessed on 7 July 2021).

24. Ramezani, B.; da Silva, M.G.; Simões, N. Application of smart readiness indicator for Mediterranean buildings in retrofitting actions. Energy Build. 2021, in press. [CrossRef]

25. Blanco, T.; Marco, A.; Casas, R. Online social networks as a tool to support people with special needs. Comput. Commun. 2016, 73, 315-331. [CrossRef]

26. Blanco, T.; Casas, R.; López-Pérez, J.M. Common Understanding Area (CUA): An x-disciplinary design tool for technological innovation. In Proceedings of the Technologies Applied to Electronics Teaching Conference (IEEE TAEE), La Laguna, Spain, 20-22 June 2018; pp. 1-8.

27. Stickdorn, M.; Schneider, J. This Is Service Design Thinking: Basics, Tools, Cases; Wiley: Hoboken, NJ, USA, 2011.

28. Testo 480 Certified Calibration Device. 2021. Available online: https:/ / www.testo.com/es-ES/instrumento-multi-parametrotesto-480/p/0563-4800 (accessed on 7 July 2021).

29. Yao, Y.; Shekhar, D.K. State of the art review on model predictive control (MPC) in Heating Ventilation and Air-conditioning (HVAC) field. Build. Environ. 2021, 200, 107952. [CrossRef]

30. Adelantado, F.; Vilajosana, X.; Tuset-Peiro, P.; Martinez, B.; Melia-Segui, J.; Watteyne, T. Understanding the Limits of LoRaWAN. IEEE Commun. Mag. 2017, 55, 34-40. [CrossRef]

31. Air-Quality Parameters. Product Catalogue for Digital Industries and Smart Infrastructure. 2021. Available online: https: / / mall.industry.siemens.com/mall/en/WW/Catalog/Products/10274404\# (accessed on 7 July 2021).

32. SenseCAP Wireless CO2 Sensor LoRaWAN AU915 MHz. Features, System Architecture and Product Details. 2021. Available online: https: / /www.seeedstudio.com/SenseCAP-LoRaWAN-CO2-Sensor-LoRaWAN-AU915-p-4975.html (accessed on 7 July 2021).

33. Aranet 4 Pro. Specifications and Technical Documentation. 2021. Available online: https://aranet.com/product/aranet4-sensor/ (accessed on 7 July 2021).

34. ISO 50001:2018 Standard. Energy Management Systems. Requirements with Guidance for Use. 2018. Available online: https:/ / www.iso.org/obp/ui/es/\#iso:std:iso:50001:ed-2:v1:es (accessed on 7 July 2021).

35. Siemens PAC3200 Power Meter. Functional Features. 2021. Available online: https://www.siemens.com/download?BTLV_50409 (accessed on 7 July 2021). 
36. Spanish Government. Technical Building Code (CTE). 2017. Available online: https://www.codigotecnico.org/DocumentosCTE/ AhorroEnergia.html (accessed on 7 July 2021).

37. IDEA Study. Sectorial Energy Monitoring. Educational Centers: Universities. 2012. Available online: https://www.idae.es/ uploads/documentos/documentos_IDAE_Memoria_Anual_2012_08fa77c5.pdf (accessed on 7 July 2021). 